\title{
The Working Process and Time Efficiency of Patient Transportation in Cardiovascular Hospital Using Time Process Modeling
}

\author{
Hiroko Ojima ${ }^{1}$, Yuko Ohno ${ }^{1}$, Sachiko Shimizu ${ }^{1}$, Shintaroh Oi ${ }^{1}$, Yasuko Inoue ${ }^{1}$, \\ Atsue Ishii ${ }^{1}$, Satoko Kasahara ${ }^{2}$, Katsumi Hirakawa ${ }^{3}$, Shohei Nakamura ${ }^{4}$, \\ Ichiro Kanaya ${ }^{4}$, Kazuo Kawasaki ${ }^{4}$, Atsuko Tanaka ${ }^{5}$, \\ Fujie Motosugi ${ }^{5}$, and Chizuru Okada ${ }^{5}$ \\ ${ }^{1}$ School of Medicine, Osaka University, Yamadaoka1-7, Suita, Osaka, 5650871 Japan \\ ${ }^{2}$ School of Medicine, Kochi University, Nankoku-shi, Kochi, 783-0043 Japan \\ ${ }^{3}$ School of Human Nursing, University of Shiga Prefecture, 2500 Yasaka, \\ Hikone, Shiga, 522-8573, Japan \\ ${ }^{4}$ School of Engeneering, Osaka University, Yamadaoka2-1, Suita, 5650871 Osaka, Japan \\ ${ }^{5}$ National Cardiovascular Center, 5-7-1 Fujishiro-dai, Suita, Osaka 565-8565, Japan \\ \{h.noda, ohno, shimizu, atsue\} @sahs.med.osaka-u.ac.jp, \\ \{nakamura, kanaya\}@design.frc.eng.osaka-u.ac.jp, \\ s-kasahara@kochi-u.ac.jp, khirakawa@nurse.usp.ac.jp, \\ \{tanakaa, fmotosug, tokada\}@hsp.ncvc.go.jp
}

\begin{abstract}
Patient transportation is one of the daily and frequent jobs in the hospital, however, it requires much strain and time of nurses. We carried out continuous-observation time and motion study (TMS) on the second time scale with recording by the other recorder in four wards of a cardiovascular disease hospital. Based on the recorded data, we carried out time processes modeling (TPM), that visualize the each transportation process sketchy and we could investigate the workflows of transportation as event instance.
\end{abstract}

Keywords: patient-transportation, Time Process Modeling, work process.

\section{Introduction}

Patient transportation is crucial issue in nursing care from the view points of both quality assurance and patient safety. In addition, the burden of the patient transportation service on nurses is both physically and mentally high and operation management is also important on the ward.

In this study, we analyze the patient transportation in the cardiovascular disease hospital to consider the process of transfer in the case of a complex situation and condition. We propose a unified modeling language (UML) based workflow modeling in order to capture the work process of the patient transportation. We describe the patient transportation prototype in the acute care hospital and propose some solutions to optimize workflow. 


\section{Materials and Methods}

We conducted an observational, time and motion study in four wards. A continuous observation time study was conducted from March 9 to March 13,2009, in four different wards; $10^{\text {th }}$ (department of cerebral vascular disease), $8^{\text {th }}$ (department of valvular disease cardiomyopathy pulmonary hypertension), $7^{\text {th }}$ western (department of cardiac dysrhythmia valvular disease cardiomiopathy) and $7^{\text {th }}$ east (department of cardiac dysrhythmia valvular disease cardiac failure).

Based on time and motion studyrecords, we modeled workprocessby the time process study, expanded method for work flow and process analysis. TPM clarify the business process and analyzing the process of task, And, we apply the display technique of the UML, UML's Activity like diagram and use case like diagram help to model the detailed logic of care process.

Finally, we listed up each activity's attribute and time (named Event List). Event List is the list of work actions and is basis for structuring the business. We listed up the actors and resources for each event. And based on Time Motion Study data, we set up amount of time required.

\section{Result and Conclusion}

The twenty-two transportations were occurred inside the ward, and average required time is 0:05:46. Of the 22, eight diagrams have exactly the same work flow. There is no same flow diagram as Fig1. Required time for example1 is 0:02:10.

Number of diagrams modeling the outside the ward transportation are eighteen, and average required time is 0:14:40. Each diagram is different.

There are 40 events with patient transportation. There are two kinds of the actors: nurse, nurse's aid, and 5 kinds of the human resource: other nurse, contact personnel, doctor, patient, patient's

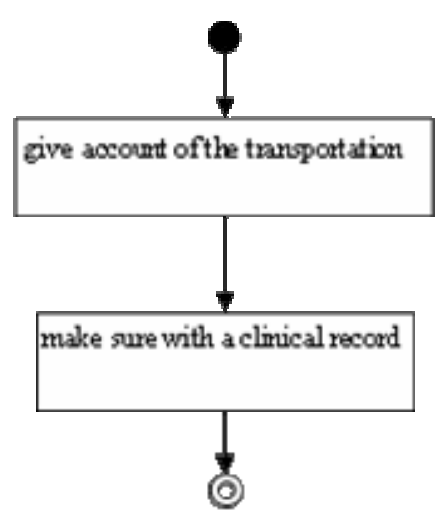

Fig. 1. Example (inside the ward) family and 16 kinds of the object resource: telephone, wheelchair, gurney, clinical record, identification card, electrocardiogram, drip, urine bag, personal computer, thermometer, blood pressure meter, elevator, chair, weight scale, X-ray picture and 2 kinds of the information resource: transport information, patient information.

As the result of this study, we find different process by ward. We also clarified that the work time was affected by transport type, ward characteristics and patient age. 